\title{
65. Effective Stimulation Parameters for the LTP Formation in the Superior Colliculus Slices from the Guinea Pig
}

\author{
By Takaaki MiYamoto and Yasuhiro OKADA \\ Department of Physiology, Kobe University School of Medicine, \\ Chuo-ku, Kobe 650 \\ (Communicated by Yasuji KatsukI, M. J. A., Sept. 12, 1988)
}

In 1973, Bliss and LQmo found the long-term potentiation (LTP) in the hippocampus of rabbits which was maintained for several hours after a short period of tetanic stimulation. ${ }^{1)}$ While LTP can now be easily demonstrated, the distribution of LTP in the brain, the mechanism underlying the phenomenon, and the functional significance of the LTP remain unclear. ${ }^{2)}$ However, LTP formation is interpreted as substantial increase in synaptic efficacy and so attracts great interest because of the possibility that the phenomenon might underlie some aspects of memory storage. ${ }^{3)}$ It was suggested that LTP might be a general synaptic plasticity for modifiable synapses. If that is so, it is expected that LTP can be reliably recorded in many parts of the central and peripheral nervous systems, including the superior colliculus (SC).

Over the past 15 years, research findings on the formation of LTP in the mammalian brain have mainly come from the hippocampus and the neocortex area. LTP formation in the superior colliculus has not been reported in the literature except for goldfish tectum. ${ }^{4}$ )

In this experiment, we reported the first demonstration of LTP formation in the SC slice from the guinea pig and determined the optimal stimulation parameters for the LTP formation.

Method. Guinea pigs weighing between 200 and $300 \mathrm{~g}$ (one month old) were decapitated, and the brains quickly removed. Tissue blocks of SC were rapidly dissected out and cut sagittally into slices of between 400 and $600 \mu \mathrm{m}$ thickness with a razor blade. Details of the slice preparation have been reported elsewhere. ${ }^{5}$ ) Before beginning the experiments, the slices were preincubated for a minimum of $20 \mathrm{~min}$ in the standard medium (concentration in $\mathrm{mM}: \mathrm{NaCl} 125$, $\mathrm{KCl} 3, \mathrm{KH}_{2} \mathrm{PO}_{4} 1.25, \mathrm{CaCl}_{2} 2, \mathrm{MgSO}_{4} 1.3, \mathrm{NaHCO}_{3} 26, \mathrm{D}$-glucose 10) equilibrated with $95 \% \mathrm{O}_{2}$ and $5 \% \mathrm{CO}_{2}(\mathrm{pH} \mathrm{7.4)}$. Thereafter each slice was transferred to the recording chamber under a stereomicroscope and submerged completely in the perfusion medium. Each layer of the SC can be clearly visualized and identifiable under the stereomicroscope. The chamber was perfused continuously with the standard medium at a rate of $8 \mathrm{ml} / \mathrm{min}$. The temperature in the chamber was kept at $35.5^{\circ} \mathrm{C}$ throughout the experiment. Glass microelectrode filled with $2 \mathrm{M}$ $\mathrm{NaCl}$ was placed in the superficial grey layer (SGL) of the SC and bipolar silver wire electrode for stimulation was placed in the optic layer (OL). Square pulses of $100 \mu \mathrm{sec}$ in duration (Stimulator, Nihon Koden SEN7103) were applied for test stimulation which were discharged at the rate of $0.5 \mathrm{~Hz}$. Electrical activity was recorded by oscilloscope (Nihon Koden VC10). The evoked field potential recorded here has been reported to be postsynaptic in nature.5)

Results. Fig. 1 shows a typical example of the LTP formation in the SGL. 


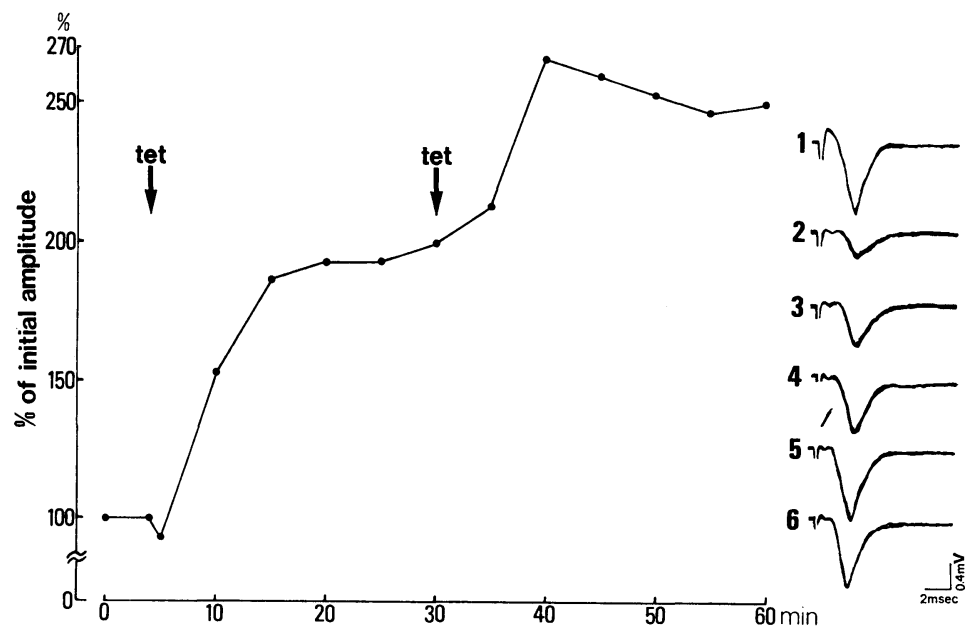

Fig. 1. The appearance of LTP in SGL of SC slice and the time course of a typical example of the LTP formation. Right panel shows the PSPs elicited in the SGL of SC slice after the stimulation to OL. (1) indicates the PSP of maximum amplitude with one test stimulus. In (2) the stimulus intensity was adjusted to evoke PSP for the amplitude to be about $1 / 3$ of the maximum amplitude. (3) and (4) show potentiated PSPs 5 and $15 \mathrm{~min}$ after the tetanic stimulation $(50 \mathrm{~Hz}$, $20 \mathrm{sec}$ ), respectively. Furthermore (5) and (6) show more potentiated PSPs 10 and $20 \mathrm{~min}$ after the second tetanic stimulation, respectively. Left panel indicates the time course of LTP formation of the slice shown in the right panel. In the figure the adjusted amplitude of PSP in (2) of right panel was taken as $100 \%$. At 'tet $\downarrow$ ', the tetanic stimulation was applied to OL.

By controlling the intensity of the stimulus, maximum amplitude of PSP was first noted ( 1 of right-hand panel in Fig. 1) and then the intensity of the stimulation was adjusted for the PSP amplitude to be about $1 / 3$ of the maximum amplitude (2 of right-hand panel) before the experiments were started. Here we employed the tetanic stimulation at the frequency $50 \mathrm{~Hz}$ and $20 \mathrm{sec}$ in duration and this adjusted amplitude of PSP was regarded as $100 \%$. The PSP was increased to about $190 \%$ of initial amplitude $15 \mathrm{~min}$ after tetanic stimulation (4 of right-hand panel). Furthermore, second tetanic stimulation was delivered 25 min after the first tetanus. PSP was increased further to about $270 \%$ of initial amplitude $10 \mathrm{~min}$ after the second stimulation ( 5 of right-hand panel). Thereafter PSP was maintained in about $250 \%$ of initial amplitude ( 6 of righthand panel). The left panel shows a time course of the LTP formation recorded from this preparation using $50 \mathrm{~Hz}$ and $20 \mathrm{sec}$ tetanic stimulation.

The formation of LTP can be modified by the stimulation parameters such as frequency, duration, and intensity. ${ }^{2)}$ In this experiment, we adjusted the intensity of the stimulation so as to evoke the PSP at about $1 / 3$ of the maximum amplitude which empirically proved most potentiated for the LTP formation. In our SC slice preparation LTP was not observed at the frequency of less than $30 \mathrm{~Hz}$ or more than $200 \mathrm{~Hz}$. Fig. 2 represents the time course of the LTP formation in the $\mathrm{SC}$ slices stimulated for the 1,10 , and $20 \mathrm{sec}$ in duration at $100 \mathrm{~Hz}$ (A) and for the $1,10,20$, and $30 \mathrm{sec}$ at $50 \mathrm{~Hz}$ (B), respectively. It can be seen from Fig. 2 that the PSP amplitude was increased to $178 \%$ of the control 

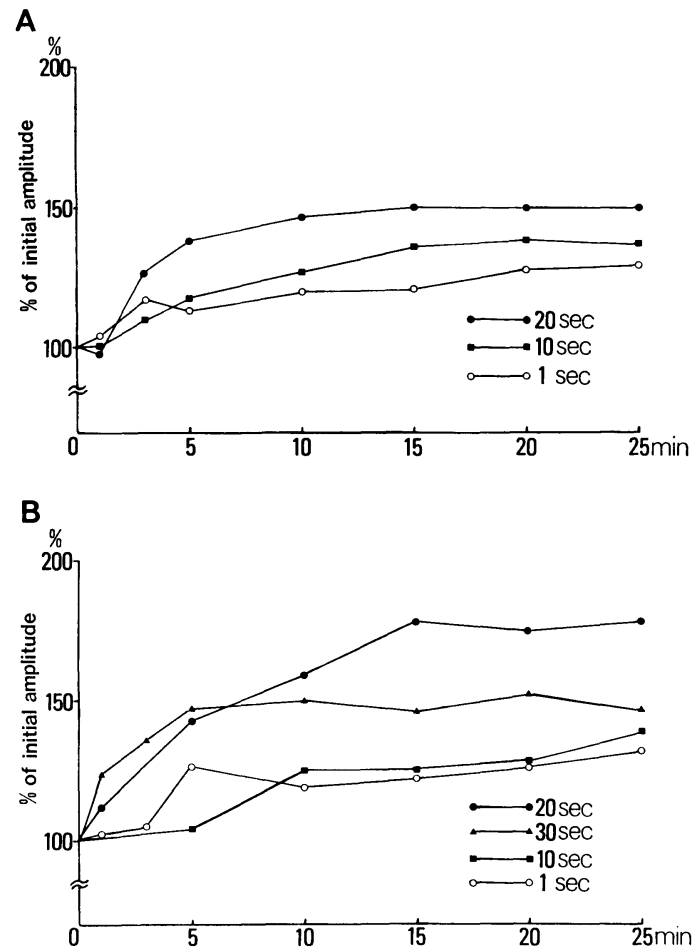

Fig. 2. The occurrence of LTP after the tetanic stimulations at the frequency of $100 \mathrm{~Hz}$ and $50 \mathrm{~Hz}$ in different duration. In (A), tetanic stimulation at the frequency of $100 \mathrm{~Hz}$ was applied for 1 ( $\bigcirc-\bigcirc), 10(\mathbf{\square - n})$, and $20 \mathrm{sec}$ (-) in duration. In (B), tetanus at the frequency of $50 \mathrm{~Hz}$ was applied for $1(\bigcirc-\bigcirc), 10(\boldsymbol{\square}-\boldsymbol{\square}), 20(\bullet-\bullet)$, and $30 \mathrm{sec}(\boldsymbol{\Delta}-\mathbf{\Delta})$. Each plot indicates the average value of five slices. Standard error means for each plot are not indicated in the figure, but they were within $15 \%$ of the mean value.

value $15 \mathrm{~min}$ after tetanic stimulation at $50 \mathrm{~Hz}$ and $20 \mathrm{sec}$ in duration and it was stabilized at this level thereafter. LTP formation was not so distinctly induced by the tetanic stimulation of 1 and $10 \mathrm{sec}$ in duration. The rate of LTP formation was thus maximum for the tetanic stimulation of $50 \mathrm{~Hz}$ and $20 \mathrm{sec}$ in duration.

Discussion. In the present experiment, the postsynaptic potential recorded in the SGL after the OL stimulation must be postsynaptic in nature ${ }^{5)}$ and elicited by the activation of retino-tectal pathway because the same type of potential could be recorded after the stimulation of optic nerve (figure not shown). The finding that LTP can be reliably recorded in the SC is important and significant because this is the first demonstration of this phenomenon in the SC of the mammalian brain. LTP can be induced by a wide range of tetanus frequency, intensity and duration. As for the duration of the tetanus, it has been reported in hippocampal slices that LTP was easily induced by a tetanus applied for $1 \mathrm{sec}$ at $\left.100 \mathrm{~Hz} .{ }^{2}\right)$ Yamamoto and Sawada indicated that the duration of the tetanus was not an important parameter if it was larger than $1 \mathrm{sec}$ in hippocampal slices. ${ }^{6)}$ In hippocampal slices a total of 100-200 pulses are required for inducing LTP and furthermore at lower frequencies of stimulation (below 
$50 \mathrm{~Hz}$ ) a heterosynaptic response depression can be seen following the termination of the tetanus, ${ }^{7}$ ) and at higher frequencies of stimulation (between 100 and $400 \mathrm{~Hz}$ ) such heterosynaptic depression is rarely observed. ${ }^{8)}$ On the contrary, our experiments of SC slice indicated that the tetanic frequency of $50 \mathrm{~Hz}$ and $20 \mathrm{sec}$ in duration was most effective for the LTP formation and a total of 1000 pulses were required for the formation of distinct LTP.

It has been reported that the NMDA receptor, a subtype of glutamate receptor may be involved in the formation of LTP.2),9) For testing this possibility in SC slice, we studied the effect of DL-2-amino-5-phosphonovalerate (APV), a specific antagonist against NMDA receptor, in order to know whether or not it might inhibit the appearance of LTP in SC. In the preparation of SC, a large dose of APV $(500 \mu \mathrm{M})$ inhibited the formation of LTP, showing that NMDA receptor may also be involved in the LTP phenomenon in SC. In the present study, the distinct LTP formation was observed in the SGL of SC. The details of mechanism of LTP formation in SC, however remain to be elucidated by further studies.

\section{References}

1) Bliss, T. V. P., and Lфmo T. J.: Physiol., 232, 331-356 (1973).

2) Teyler, T. J., and DiScenna, P.: Ann. Rev. Neurosci., 10, 131-161 (1987).

3) Squire, L.: ibid., 5, 241-273 (1982).

4) Lewis, D., and Teyler, T.: Brain Res., 375, 246-250 (1986).

5) Arakawa, T., and Okada, Y.: Proc. Japan Acad., 63B, 389-392 (1987).

6) Yamamoto, C., and Sawada, S.: Exp. Neurology, 74, 122-130 (1981).

7) Alger, B. E., Megela, A. L., and Teyler, T. J.: Brain Res. Bull., 3, 181-184 (1978).

8) McNaughton, B. L., Douglas, R. M., and Goddard, G. V.: Brain Res., 157, 277-293 (1978).

9) Morris, R. G. M. et al.: Nature, 319, 774-776 (1986). 